University of Texas at El Paso

ScholarWorks@UTEP

$3-2020$

\title{
How to Combine (Dis)Utilities of Different Aspects into a Single (Dis)Utility Value, and How This Is Related to Geometric Images of Happiness
}

Laxman Bokati

The University of Texas at El Paso, Ibokati@utep.edu

Nguyen Hoang Phuong

Thang Long University, nhphuong2008@gmail.com

Olga Kosheleva

The University of Texas at El Paso, olgak@utep.edu

Vladik Kreinovich

The University of Texas at El Paso, vladik@utep.edu

Follow this and additional works at: https://scholarworks.utep.edu/cs_techrep

Part of the Computer Sciences Commons

Comments:

Technical Report: UTEP-CS-20-20

To appear in Journal of Advanced Computational Intelligence and Intelligent Informatics (JACIII)

\section{Recommended Citation}

Bokati, Laxman; Phuong, Nguyen Hoang; Kosheleva, Olga; and Kreinovich, Vladik, "How to Combine (Dis)Utilities of Different Aspects into a Single (Dis)Utility Value, and How This Is Related to Geometric Images of Happiness" (2020). Departmental Technical Reports (CS). 1422.

https://scholarworks.utep.edu/cs_techrep/1422

This Article is brought to you for free and open access by the Computer Science at ScholarWorks@UTEP. It has been accepted for inclusion in Departmental Technical Reports (CS) by an authorized administrator of ScholarWorks@UTEP. For more information, please contact Iweber@utep.edu. 


\title{
How to Combine (Dis)Utilities of Different Aspects into a Single (Dis)Utility Value, and How This Is Related to Geometric Images of Happiness
}

\author{
Laxman Bokati ${ }^{1}$, Hoang Phuong Nguyen ${ }^{4}$, \\ Olga Kosheleva ${ }^{2}$, and Vladik Kreinovich ${ }^{1,3}$ \\ ${ }^{1}$ Computational Science Program \\ ${ }^{2}$ Department of Teacher Education \\ ${ }^{3}$ Department of Computer Science \\ University of Texas at El Paso \\ $500 \mathrm{~W}$. University \\ El Paso, TX 79968, USA \\ lbokati@utep.edu,vladik@utep.edu \\ ${ }^{4}$ Division Informatics, Math-Informatics Faculty \\ Thang Long University \\ Nghiem Xuan Yem Road \\ Hoang Mai District \\ Hanoi, Vietnam, nhphuong2008@gmail.com
}

\begin{abstract}
In many practical situations, a user needs our help in selecting the best out of a large number of alternatives. To be able to help, we need to understand the user's preferences. In decision theory, preferences are described by numerical values known as utilities. It is often not feasible to ask to user to provide utilities of all possible alternatives, so we must be able to estimate these utilities based on utilities of different aspects of these alternatives. In this paper, we provide a general formula for combining utilities of aspects into a single utility value. The resulting formula turns out to be in good accordance with the known correspondence between geometric images and different degrees of happiness.
\end{abstract}

\section{Formulation of the Problem}

Sometimes, we need help when making a decision. In many practical situations, we need help when making decisions. One of the reasons for this need is that while we can meaningfully compare a few alternatives, in real life, 
there is often a huge number of alternatives, so huge that it is not realistic to consider them all.

In such situations, a natural idea is to use a computer-based system to help us make decisions.

\section{What a computer-based system needs to do.}

- This system will first try to understand what exactly we want, by asking us to compare different alternatives.

- Then, the system will use this knowledge to predict how we will react to other possible alternatives - and select an alternative that we would (hopefully) prefer.

How exactly will the system do it. Every alternative can be characterized by several aspects. For example, when we decide which apartment to rent, we take into account the amount of money that we need to pay, the apartment's size, its distance from our workplace (and/or from our friends), on what floor is this apartment, etc. In a big city, there are usually many apartments, with many possible combinations of these aspects. It is not realistic to expert the user to provide an opinion on all possible combinations of such aspects.

What we can do is:

- we can elicit a person's opinion about each of these aspects, and then

- we can try to find the way of combining these opinions which closely resembles how this particular person makes decisions.

How do we describe user's preferences: decision theory's utility-based approach. In decision theory, user's preferences are usually described by a number called utility; see, e.g., [3, 7, 10, 11, 12].

Usually, for each aspect, we can describe the worst possible situation $A_{-}$ among those that we can still consider - and we can describe the ideal situation $A_{+}$. Usually:

- the worst possible - but still acceptable - situation is easy to describe: e.g., for apartment's area, it is 10 square meters (there are actually such mini-apartments in Hong Kong), while

- the same user can provide us with different ideal situations: 200 square meters? a million square meters? the only limit to this is the flight of fantasy.

Both situations $A_{-}$and $A_{+}$are extreme. To describe the user's opinion about each intermediate situation $A$, we can ask the user to compare this situation $A$ with lotteries $L(p)$, in which the user will:

- get the ideal situation $A_{+}$with probability $p$, and

- get the worst possible situation $A_{-}$with the remaining probability $1-p$. 
When $p$ is close to 1 , the lottery is almost as good as getting the ideal situation $A_{+}$, so most probably the user will prefer this lottery to a less glamorous alternative $A$. We will denote this preference by $A<L(p)$.

Vice versa, when $p$ is close to 0 , the lottery is almost as bad as the worst alternative $A_{-}$, so we expect that $L(p)<A$.

One can easily see that, as we increase $p$ from 0 to 1 , there will be threshold value when $L(p)<A$ is replaced by $A<L(p)$, i.e., the value

$$
u(A)=\sup \{p: L(p)<A\}=\inf \{p: A<L(p)\} .
$$

This threshold value is called the utility of the alternative $A$.

By definition, this value has the property that for each $\varepsilon>0$, we have $L(u(A)-\varepsilon)<A<L(u(A)+\varepsilon)$. For very small $\varepsilon>0$, probabilities $u(A)-\varepsilon$ and $u(A)+\varepsilon$, and thus, the corresponding lotteries are practically indistinguishable. Thus, from the practical viewpoint, the alternative $A$ is equivalent to the lottery $L(u(A))$. We will denote this equivalence by $A \equiv L(u(A))$.

Important: utility is defined modulo a multiplicative constant. As we have mentioned earlier:

- while we can usually uniquely determine the worst alternative $A_{-}$,

- we can select different alternatives as ideal ones.

What if we replace the original "ideal" alternative $A_{+}$with a slightly worse (but still ideal) alternative $A_{+}^{\prime}$ ? How will this affect the numerical values of utility?

The fact that the new ideal situation $A_{+}^{\prime}$ is somewhat worse than the original ideal situation $A_{+}$, i.e., that $A_{-} \leq A_{+}^{\prime}<A_{+}$means that we can find its utility $u\left(A_{+}^{\prime}\right)$ in the original $A_{+}$-based scale. So, the new ideal situation $A_{+}^{\prime}$ is equivalent to the lottery $L\left(u\left(A_{+}^{\prime}\right)\right)$, in which:

- we get $A_{+}$with probability $u\left(A_{+}^{\prime}\right)$, and

- we get $A_{-}$with the remaining probability $1-u\left(A_{+}^{\prime}\right)$.

Suppose that in the new $A_{+}^{\prime}$-based scale, an alternative $A$ has utility $u^{\prime}(A)$. This means that the alternative $A$ is equivalent to a lottery $L^{\prime}\left(u^{\prime}(A)\right)$ in which:

- we get $A_{+}^{\prime}$ with probability $u^{\prime}(A)$, and

- we get $A_{-}$with the remaining probability $1-u^{\prime}(A)$.

As we have mentioned, the alternative $A_{+}^{\prime}$ is, in its turn, equivalent to a lottery $L\left(u\left(A_{+}^{\prime}\right)\right)$. Thus, the alternative $A$ is equivalent to a two-stage lottery, in which:

- first, we select $A_{+}^{\prime}$ with probability $u^{\prime}(A)$ and $A_{-}$with the remaining probability $1-u^{\prime}(A)$, and then

- if we selected $A_{+}^{\prime}$ on the first stage, we select $A_{+}$with probability $u\left(A_{+}^{\prime}\right)$ and $A_{-}$with the remaining probability $1-u\left(A_{+}^{\prime}\right)$. 
As a result of this two-stage lottery, we get either $A_{+}$or $A_{-}$, and the probability to get $A_{+}$is equal to $c \cdot u^{\prime}(A)$, where we denoted $c \stackrel{\text { def }}{=} u\left(A_{+}^{\prime}\right)$. By definition, this probability is exactly the utility $u(A)$ in the $A_{+}$-based scale. So we conclude that

$$
u(A)=c \cdot u^{\prime}(A) .
$$

In mathematical terms, we can say that utility is defined modulo multiplication a constant factor.

Towards the formulation of the problem. Our original problem is that:

- we know the preferences corresponding to each aspect, and

- we need to combine these preferences, so that we will be able to compare different alternatives - each of which is characterized by different values of different aspects.

As we have mentioned earlier, in decision theory, preferences are described by utilities. In terms of the utilities, the above problem can be formulated as follows:

- we know utilities $u_{1}, \ldots, u_{n}$ corresponding to all $n$ aspects, and

- based on these values, we need to estimate the overall utility $u$ of the alternative.

\section{Analysis of the Problem and the Resulting Al- gorithm}

What do we want? We want an operation $f(a, b)$ that would combine two utility values $a \geq 0$ and $b \geq 0$ into a combined value $f(a, b) \geq 0$.

We want this operation to be general, to be able to combine every two aspects. In particular, if you swap the aspects, i.e., if what was the first aspect will now be the second one and vice versa, we should get the same combination result, i.e., we should have $f(a, b)=f(b, a)$. In mathematical terms, this means that the combination operation must be commutative.

If we need to combine utility values $a, b$, and $c$ corresponding to three aspects, then:

- we can first combine $a$ and $b$ into $f(a, b)$, and then add $c$ to this combination, resulting in $f(f(a, b), c)$;

- alternatively, we can first combine $b$ and $c$ into $f(b, c)$, and then add $a$ to this combination, resulting in $f(a, f(b, c))$.

It is reasonable to require that these two approaches lead to the same estimate, i.e., that we have $f(f(a, b), c)=f(a, f(b, c))$. In mathematical terms, this means that the combination operation must be associative. 
If one of the aspects become better, and the other remains the same, then the overall utility should increase: if $a<a^{\prime}$, then we should have $f(a, b)<f\left(a^{\prime}, b\right)$. In mathematical terms, this means that the combination operation must be strictly monotonic.

Finally, since utility is defined modulo a multiplicative factor, the combination result should be the same if we re-scale all the values, i.e., if $f(a, b)=v$ then for each $c>0$, we should have $f(c \cdot a, c \cdot b)=c \cdot v$. In mathematical terms, this means that the combination operation must be scale-invariant.

Main result. The following two conditions are equivalent to each other for each function $f(a, b)$ :

- the operation $f(a, b)$ is commutative, associative, strictly monotonic, and scale-invariant;

- the operation $f(a, b)$ has the form

$$
f(a, b)=\left(a^{p}+b^{p}\right)^{1 / p}
$$

for some $p>0$.

Proof. One can easily check that every operation $f(a, b)=\left(a^{p}+b^{p}\right)^{1 / p}$ is commutative, associative, strictly monotonic, and scale-invariant.

Vice versa, let us assume that an operation $f(a, b)$ is commutative, associative, strictly monotonic, and scale-invariant. Strict monotonicity implies a weaker monotonicity property, that if $a \leq a^{\prime}$ then $f(a, b) \leq f\left(a^{\prime}, b\right)$. It is known (see, e.g., [1]) that every operation which is commutative, associative, scale-invariant, and monotonic (in the above weaker sense) is either of the form $f(a, b)=\left(a^{p}+b^{p}\right)^{1 / p}$ for some $p>0$, or has one of the three additional forms $f(a, b)=0, f(a, b)=\min (a, b)$ and $f(a, b)=\max (a, b)$. One can easily check that these three additional forms are not strictly monotonic, so the only remaining form is indeed $f(a, b)=\left(a^{p}+b^{p}\right)^{1 / p}$.

The statement is proven.

Conclusion. We thus conclude that a reasonable way to combine utilities of different aspects into a single utility value is to use the formula (1). In other words, if an alternative has $n$ aspects with utilities $u_{1}, \ldots, u_{n}$, then a reasonable estimate for its overall utility is

$$
\left(u_{1}^{p}+\ldots+u_{n}^{p}\right)^{1 / p} .
$$

Which value $p$ should we choose? In general, the parameter $p$ should be selected to match the preferences of a specific user.

In general, a reasonable idea is to select $p=2$. Indeed, in the case when each aspect can be described by its monetary value, a natural idea is to simply add these monetary values to get the overall monetary value. Empirical data shows that utility is approximately proportional to the square root of the money 
amount $u(m)=C \cdot \sqrt{m}$; see, e.g., [6] (a theoretical explanation for this formula is provided in [9]).

Since utility is defined modulo a multiplicative factor, we can re-scale utility and get $u(m)=\sqrt{m}$. So, the corresponding money amount $m$ is equal to $u^{2}$. If we add money amounts $m_{1}=u_{1}^{2}$ and $m_{2}=u_{2}^{2}$ corresponding to two aspects, we will get the overall money amount $m=m_{1}+m_{2}=u_{1}^{2}+u_{2}^{2}$ that corresponds to the utility $u=\sqrt{m}=\sqrt{u_{1}^{2}+u_{2}^{2}}$. This is exactly formula (1) for $p=2$.

Comment about disutility. In some cases, instead of good situations of looking for the best gain, we are in a not so good situation of looking for the smallest loss. Such situations can be similarly described by disutility - negative utility - and a similar formula can be similarly derived for combining disutilities of different aspects into a single value.

\section{How Is This Related to Geometric Images of Happiness}

What is the consequence of using different values $p$ when combining disutilities. Ideally, we want to select an alternative that minimized disutility. If we cannot perform an exact minimization, then at least we should select an alternative for which the overall disutility is smaller than a certain pre-defined value $u_{0}$.

In view of the above formula, this means selecting an alternative for which

$$
\left(u_{1}^{p}+\ldots+u_{n}^{p}\right)^{1 / p} \leq u_{0} .
$$

What happens when we change $p$ ? It is known that the expression

$$
\left(u_{1}^{p}+\ldots+u_{n}^{p}\right)^{1 / p}
$$

decreases as $p$ increases: if $p<p^{\prime}$, then

$$
\left(u_{1}^{p^{\prime}}+\ldots+u_{n}^{p^{\prime}}\right)^{1 / p^{\prime}}<\left(u_{1}^{p}+\ldots+u_{n}^{p}\right)^{1 / p} .
$$

Thus, if an alternative with certain values of $u_{1}, \ldots, u_{n}$ was acceptable for some value $p$, it is will be acceptable for all larger values $p^{\prime}>p$.

So, the larger $p$, the more alternative are acceptable - and thus, the more choices a person has.

- When $p$ is very small, few alternatives are acceptable - in other words, the situation is very gloomy.

- On the other hand, when $p$ increases, more and more alternatives will become acceptable - and thus, the whole situation will feel less and less gloomy. 
From this viewpoint, the value $p$ can be viewed as a measure of a person's satisfaction with a situation, as a measure of - if you want - happiness (please take into account that we are talking about avoiding losses, so happiness is a relative term here).

Geometric images of happiness. Interestingly, according to $[2,4,5,8]$, a proper geometric image of happiness is exactly the 2 -D version of the above set: the set of all the points $\left(u_{1}, u_{2}\right)$ for which $\left(u_{1}^{p}+u_{2}^{p}\right)^{1 / p} \leq u_{0}$. Out of these sets:

- sets with larger $p$ correspond to larger degree of happiness, while

- sets corresponding to $p \approx 0$ correspond to unhappiness.

Thus, our analysis provides a theoretical explanation for this empirical fact.

\section{Acknowledgments}

This work was supported in part by the US National Science Foundation grants 1623190 (A Model of Change for Preparing a New Generation for Professional Practice in Computer Science) and HRD-1242122 (Cyber-ShARE Center of Excellence).

The authors are thankful to all the participants of the International Conference on Artificial Intelligence and Computational Intelligence AICI'2020 (Hanoi, Vietnam, January 4-6, 2020) for valuable discussions.

\section{References}

[1] K. Autchariyapanitkul, O. Kosheleva, V. Kreinovich, and S. Sriboonchitta, "Quantum econometrics: how to explain its quantitative successes and how the resulting formulas are related to scale invariance, entropy, and fuzziness", In: V.-N. Huynh, M. Inuiguchi, D.-H. Tran, and T. Denoeux (eds.), Proceedings of the International Symposium on Integrated Uncertainty in Knowledge Modelling and Decision Making IUKM'2018, Hanoi, Vietnam, March 13-15, 2018.

[2] F. Y. Dong and K. Hirota, "Concept of fuzzy atmosfield and its visualization", In: R. Seising, E. Trillas, C. Moraga, and S. Termini, On Fuzziness: A Homage to Lotfi A. Zadeh, Springer, 2013, Vol. 1, pp. 257-263.

[3] P. C. Fishburn, Utility Theory for Decision Making, John Wiley \& Sons Inc., New York, 1969.

[4] J. A. Garcia-Sanchez, K. Ohnishi, A. Shibata, F. Y. Dong, and K. Hirota, "Visualization method of emotion information for long distance interaction," Proceedings of the Joint 7th International Conference on Humanoid, Nanotechnology, Information Technology, Communication and Control, 
Environment and Management IEEE-HNICEM'2014 and the 6th International Conference on Computational Intelligence and Intelligent Informatics ISCIII'2014, Palawan, Philippines, November 12-16, 2014, Paper DSP09.

[5] K. Hirota, F. Dong, and J. A. Garcia Sanchez, "Multiagent smart communication based on CI technology", In: V. Kreinovich and Nguyen Hoang Phuong (eds.), Soft Computing for Biomedical Applications and Related Topics, Springer Verlag, to appear.

[6] D. Kahneman, Thinking, Fast and Slow, Farrar, Straus, and Giroux, New York, 2011.

[7] V. Kreinovich, "Decision making under interval uncertainty (and beyond)", In: P. Guo and W. Pedrycz (eds.), Human-Centric Decision-Making Models for Social Sciences, Springer Verlag, 2014, pp. 163-193.

[8] Z. T. Liu, M. Wu, D. Li, L. F. Chen, F. Y. Dong, Y. Yamazaki, and K. Hirota, "Concept of fuzzy atmosfield for representing communication atmosphere and its application to humans-robots interaction", Journal of Advanced Computational Intelligence and Intelligent Informatics JACIII, 2013, Vol. 17, No. 1, pp. 3-17.

[9] J. Lorkowski and V. Kreinovich, "Granularity helps explain seemingly irrational features of human decision making", In: W. Pedrycz and S.-M. Chen (eds.), Granular Computing and Decision-Making: Interactive and Iterative Approaches, Springer Verlag, Cham, Switzerland, 2015, pp. 1-31.

[10] R. D. Luce and R. Raiffa, Games and Decisions: Introduction and Critical Survey, Dover, New York, 1989.

[11] H. T. Nguyen, O. Kosheleva, and V. Kreinovich, "Decision making beyond Arrow's 'impossibility theorem', with the analysis of effects of collusion and mutual attraction", International Journal of Intelligent Systems, 2009, Vol. 24, No. 1, pp. 27-47.

[12] H. Raiffa, Decision Analysis, McGraw-Hill, Columbus, Ohio, 1997. 